\title{
论 文 祝贺过增元院士85华诞专辑
}

\section{基于䍉理论的船用锅炉构形优化}

\author{
陈林根 ${ }^{1,2^{*}}$, 冯辉君 ${ }^{1,2^{*}}$, 谢卓君 ${ }^{3}$, 唐威 ${ }^{3}$, 戈延林 ${ }^{1,2}$
}

1. 武汉工程大学热科学与动力工程研究所, 武汉 430205;

2. 武汉工程大学机电工程学院, 武汉 430205;

3. 海军工程大学动力工程学院, 武汉 430033

*E-mail: lgchenna@yahoo.com; huijunfeng@139.com

收稿日期: 2021-02-02; 接受日期: 2021-04-02; 网络版发表日期: 2021-07-30

国家自然科学基金(批准号: 51779262)资助项目

摘要基于炇理论和构形理论, 在三个主要换热部件(蒸发器、过热器和经济器)总换热面积一定的约束条件下, 以第III对流蒸发管束外径、过热器管外径和经济器管外径为优化变量, 对船用锅炉进行性能优化, 得到整体锅炉 的最优性能和最优构形. 首先, 以炇耗散率和耗功率线性加权组成的复合函数为目标进行优化, 并分析保热系 数、加权系数和换热率等初始设计参数对整体锅炉构形优化结果的影响. 结果表明: 第III对流蒸发管束无量纲 管外径、过热器无量纲管外径和经济器无量纲管外径分别存在最佳值 $1.2,1.3$ 和 1.1 , 使得复合函数取得三次最小 值 0.737 . 相比于初始设计点, 优化后整体锅炉的复合函数最高降低 $26.3 \%$, 锅炉综合性能得到显著提升. 其次, 采 用NSGA-II算法对整体锅炉进行两目标优化得到相应的Pareto前沿和锅炉最优构形，为整体锅炉的性能优化和结 构设计提供一个更为广泛的选择空间. 本文研究结果可为各种锅炉的性能优化设计提供理论指导.

关键词炽理论, 构形理论, 船用锅炉, 炇耗散率, 耗功率, 多目标优化

\section{1 前言}

蒸汽动力是现代船舶动力的一种重要形式, 蒸汽 锅炉是其核心部件之一. 随着锅炉技术水平的不断提 高, 出现了体积小、重量轻、经济性能高的增压锅炉. 增压锅炉内部存在着各种传热过程，对其进行分析和 优化将有助于提高锅炉的性能. 一些学者从传热特性 和流动特性入手，用传统方法对船用增压锅炉进行了 性能分析与优化.

构形理论 ${ }^{[111]}$ 既可揭示自然界各类流动系统结构 形成的机理，又可指导工程界各类传热传质过程及装
置的优化设计. 一些学者也将构形理论应用于锅炉部 件和整体系统的优化设计中. Xie等人 ${ }^{[12]}$ 基于构形理 论, 综合考虑了蒸发器的换热量和烟气流动压降, 在 蒸发器总换热面积一定的约束条件下，对增压锅炉蒸 发器进行了优化设计, 得到了复合函数最小的蒸发管 束最优构形. Xie等人 ${ }^{[13]}$ 进一步以换热率和耗功率组 成的复合函数为目标优化了该锅炉过热器结构, 优化 后过热器的耗功率大大减小. Tang等人 ${ }^{[14]}$ 采用类似方 法对锅炉经济器进行了优化, 得到了经济器最佳蛇形 管外径、管组数和管排数, 单独优化蛇形管外径、管 组数和管排数后的复合函数分别减小了 $5.7 \%, 4.9 \%$ 和

引用格式: 陈林根, 冯辉君, 谢卓君, 等. 基于㓗理论的船用锅炉构形优化. 中国科学: 技术科学, 2021, 51: 1208-1218

Chen L G, Feng H J, Xie Z J, et al. Constructal optimization for a marine boiler based on entransy theory (in Chinese). Sci Sin Tech, 2021, 51: 12081218, doi: 10.1360/SST-2021-0054 
$5.0 \%$. Gulotta等人 ${ }^{[15]}$ 应用整体性能系数法优化了生物 质锅炉换热管数目和分布, 探究了锅炉输出功率和压 降随锅炉结构的变化规律. Gulotta等人 ${ }^{[16]}$ 进一步结合 构形理论和生命周期评估法对该锅炉进行了结构优 化, 优化后锅炉的整体能耗减小了 $0.33 \%$.

清华大学过增元院士通过电热比拟方法提出了炽 理论 ${ }^{[17 ~ 26]}$, 为解决各种传热传质和热功转换过程的优 化设计 ${ }^{[27 \sim 36]}$ 提供了新的理论基础. 这一理论也被引入 到蒸汽朗肯和有机朗肯循环部件和系统的性能优化研 究中. 冯辉君等人 ${ }^{[37]}$ 应用构形理论和炇理论, 对有机工 质管壳式蒸发器进行了构形优化, 将换热器的传热积 耗散率和总洜功率进行了线性加权, 得到了最小复合 函数及对应的最佳蒸发器管外径. 冯辉君等人 ${ }^{[38]}$ 基 于炇理论, 对有机工质管壳式冷凝器进行了构形优化, 建立了同时考虑冷凝器传热和流动性能的复合函数, 得到了冷凝器的最小复合函数和最佳冷凝管外径. Wang等人 ${ }^{[27]}$ 同时考虑了朗肯循环中的熵产和䍉耗散 性能, 对循环工质质量流率进行了优化, 得到了循环各 种指标下的最优性能. 孟继安和李志信 ${ }^{[29]}$ 对 $330 \mathrm{MW}$ 电站凝汽器进行了性能分析, 发现双连树型凝汽器在 换热性能方面更具优势. Wang 等人 ${ }^{[36]}$ 对基于混合工质 的双有机朗肯循环进行了输出功率、摘产率、㶲损失 率和䍉损失率性能的综合比较, 并分析了蒸发温度、冷 凝温度和过热度等参数对循环性能的影响规律.

本文将基于䍉理论和构形理论，建立包含各传热 部件(蒸发器、过热器和经济器)的船用锅炉模型, 在 三个传热部件总换热面积一定的约束条件下开展增压 锅炉的构形优化研究, 首先以整体锅炉䛈耗散率和耗 功率线性加权组成的复合函数为优化目标, 通过优化 蒸发器、过热器和经济器管束的外径得到不同参数条 件下的最优构形, 然后采用NSGA-II算法完成炇耗散 率和耗功率两目标优化, 为实际锅炉的优化设计提供 更为全面的指导. 因此, 本文锅炉构形优化就是指在 总换热面积一定的约束条件下构建换热管最佳结构使 得锅炉性能达到最优. 这也是本文工作不同于传统锅 炉优化设计的根本之处.

\section{2 整体锅炉模型}

图1给出了船用增压锅炉模型示意图. 燃油在炉膛 燃烧后产生高温烟气, 烟气依次与蒸发器、过热器和
经济器进行热交换, 最后废气进入浴轮增压机组对烟 气涡轮做功后排出炉外. 锅炉给水依次进入经济器、 蒸发器和过热器与烟气进行热交换, 经历由未饱和 水、饱和水、饱和蒸汽和过热蒸汽的转变过程. 整个 过程中烟气和工质的换热方式近似视为顺流换热. 图2 给出了整体锅炉换热过程 $T-S$ 图. 状态点 $1 \sim 4$ 分别表示 工质侧锅炉经济器入口、经济器出口(蒸发器入口)、 蒸发器出口(过热器入口) 和过热器出口, 状态点 $5,6,9$ 和13分别表示烟气侧锅炉经济器入口、经济器出口 (蒸发器入口)、蒸发器出口(过热器入口) 和过热器出

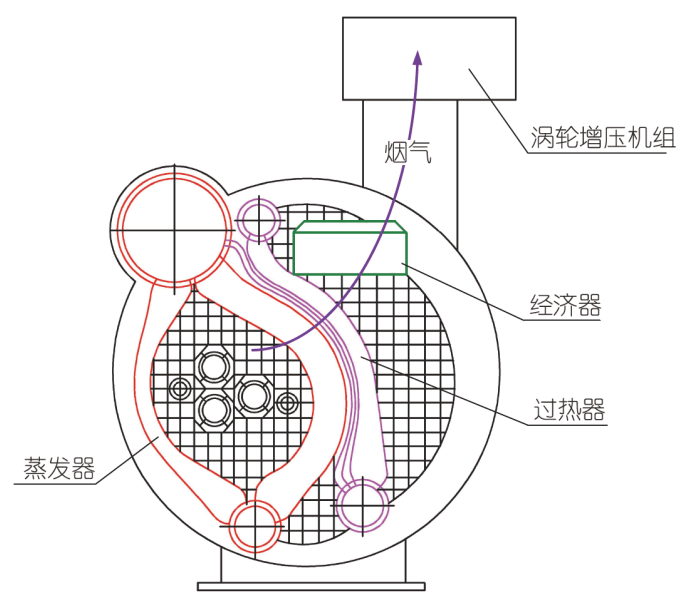

图 1 (网络版彩图)增压锅炉模型示意图

Figure 1 (Color online) Schematic diagram of the supercharged boiler.

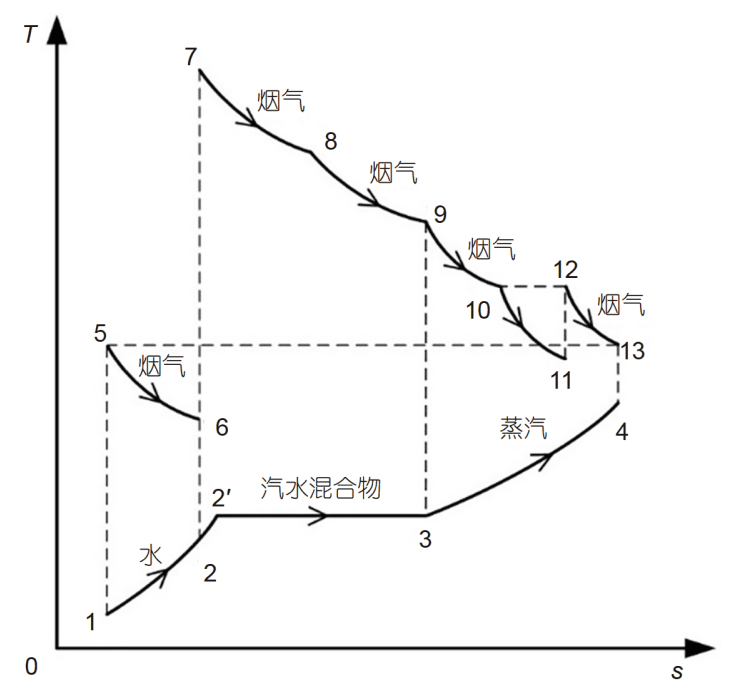

图 2 整体锅炉换热过程 $T-S$ 图

Figure $2 T-S$ diagram of the whole boiler heat exchange process. 
口, 过程 $1 \rightarrow 2,2 \rightarrow 3$ 和 $3 \rightarrow 4$ 分别表示经济器、蒸发器和 过热器的工质吸热过程, 过程 $5 \rightarrow 6,7 \rightarrow 9$ 和 $9 \rightarrow 13$ 分别 表示经济器、蒸发器和过热器的烟气放热过程. 在蒸 发器中, 状态点 8 表示炉膛出口, $7 \rightarrow 8$ 和 $8 \rightarrow 9$ 分别为烟 气在炉膛和对流蒸发管束中的放热过程. 蒸汽在过热 器中加热需经历三个流程, 状态点10 12分别表示过 热器第 1,2 流程烟气出口和第 3 流程烟气入口, $8 \rightarrow 10$, $10 \rightarrow 11$ 和 $12 \rightarrow 13$ 分别为烟气在过热器第 $1 \sim 3$ 流程中的 放热过程. 本文研究的船用增压锅炉体积很小, 但容积 热负荷很大, 同时采用浴轮增压, 这些导致本文锅炉模 型与其他锅炉模型在部件结构、布置和体积上有很大 区别.

\section{1 整体锅炉换热率}

单位时间锅炉的有效利用热量 $Q_{\mathrm{sb}}$ 为水在经济器 中吸热、水在蒸发器中蒸发和蒸汽在过热器中过热所 吸收的三部分热量总和. 因此，可得整体锅炉换热 率 $Q_{\mathrm{sb}}$ 为

$$
\begin{aligned}
Q_{\mathrm{sb}} & =Q_{\text {evap }}+Q_{\text {sht }}+Q_{\text {eco }} \\
& =\dot{m}_{\mathrm{wt}}\left(h_{3}-h_{2}\right)+\dot{m}_{\mathrm{st}}\left(h_{4}-h_{3}\right)+\dot{m}_{\mathrm{wt}}\left(h_{2}-h_{1}\right),
\end{aligned}
$$

式中, $\dot{m}_{\mathrm{wt}}$ 和 $\dot{m}_{\mathrm{st}}$ 分别为锅炉给水质量流率和过热蒸汽 产率, $h_{2}$ 和 $h_{3}$ 分别为工质在蒸发器进口和出口处的比 焓, $h_{4}$ 为工质在过热器出口处的比焓, $h_{1}$ 为工质在经济 器进口处的比焓, $Q_{\text {evap }}, Q_{\text {sht }}$ 和 $Q_{\text {eco }}$ 分别为蒸发器、过 热器和经济器的换热率. 因此, 可得锅炉的热效率 $\eta$ 为

$\eta=\frac{Q_{\mathrm{sb}}}{B Q_{\mathrm{L}}}$,

式中, $B$ 和 $Q_{\mathrm{L}}$ 分别为燃油消耗率和燃油低发热值.

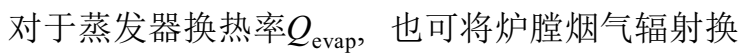
热率、水冷壁对流换热率以及对流蒸发管束换热率相 加进行计算 ${ }^{[12]}$.

$$
\begin{aligned}
Q_{\text {evap }}= & a_{\text {fur }} \sigma_{0} A_{\text {rd }}\left(T_{\mathrm{fl}}^{4}-T_{\mathrm{tw}}^{4}\right)+K_{\mathrm{con}} A_{\text {con }}\left(T_{\mathrm{fl}}-T_{\mathrm{tw}}\right) \\
& +K_{\text {cet }} A_{\text {cet }} \overline{\Delta T_{\text {cet }},}
\end{aligned}
$$

式中, $\sigma_{0}$ 为绝对黑体辐射常数, $a_{\mathrm{fur}}$ 为炉膛黑度, $A_{\mathrm{rd}}$ 为 炉膛管壁的辐射换热面积, $T_{\mathrm{fl}}$ 和 $T_{\mathrm{tw}}$ 分别为炉膛火焰温 度和管壁温度, $K_{\mathrm{con}}$ 为由烟气向管壁的对流传热系数, $A_{\text {con }}$ 为水冷壁管的对流换热面积, $K_{\text {cet }}$ 为烟气与对流蒸 发管束内工质间的传热系数, $A_{\text {cet }}$ 为对流蒸发管束换
热面积, $\overline{\Delta T_{\text {cet }}}$ 为整个换热过程中烟气与工质间的平均 温差. $A_{\text {cet }}$ 可由式(4)进行计算:

$$
A_{\text {cet }}=\pi l_{\text {cet }}\left(d_{1 \text { cet }} n_{1 \mathrm{cet}} z_{1 \mathrm{cet}}+d_{2 \mathrm{cet}} n_{2 \mathrm{cet}} z_{2 \mathrm{cet}}+d_{3 \mathrm{cet}} n_{3 \mathrm{cet}} z_{3 \mathrm{cet}}\right) \text {. }
$$

根据沿烟气流动方向对流蒸发管束管径的不同, 管束可分为 3 类, 依次称为第I, II和III对流蒸发管束. 式 (4)中, $d_{i \text { cet }}, n_{i \text { cet }}, z_{i \text { cet }}(i=1,2,3)$ 和 $l_{\text {cet }}$ 分别表示第I, II和 III对流蒸发管束管外径、管排数、单排管数和设计长 度. 对于 $Q_{\mathrm{sht}}$ 和 $Q_{\mathrm{eco}}$, 也可从烟气侧通过对流换热公式 进行计算, 详见文献[13,14,39].

\section{2 整体锅炉积耗散率}

\subsection{1 锅炉蒸发器沿耗散率}

当忽略保热系数 $\varphi$ 的影响时, 由䍉的定义可将烟气 放热过程䛈耗散率 $\dot{E}_{\phi, \mathrm{fg}}$ 表示为 ${ }^{[18]}$

$\dot{E}_{\phi, \text { fg }}=0.5 \dot{m}_{\mathrm{fg}}\left[\bar{c}_{\mathrm{p}, \text { fur }}\left(T_{\text {the }}^{2}-T_{8}^{2}\right)+\bar{c}_{\mathrm{p}, \mathrm{cet}}\left(T_{8}^{2}-T_{9}^{2}\right)\right]$.

管内工质吸热过程的䛈耗散率 $\dot{E}_{\phi, \mathrm{ww}}$ 可表示为 ${ }^{[18]}$

$$
\dot{E}_{\phi, \mathrm{sw}}=\dot{m}_{\mathrm{sw}}\left(\bar{c}_{\mathrm{p}, 2}+\bar{c}_{\mathrm{p}, 2^{2}}\right) / 2 \cdot\left(T_{2}^{2}-T_{2^{\prime}}^{2}\right) / 2+\dot{m}_{\mathrm{sw}} T_{3}\left(h_{2^{\prime}}-h_{3}\right) .
$$

忽略烟气流动过程所引起的䍉耗散率, 蒸发器总 的䍉耗散率 $E_{\phi, \text { evap }}$ 为

$$
\dot{E}_{\phi, \text { evap }}=\dot{E}_{\phi, \mathrm{fg}}+\dot{E}_{\phi, \mathrm{sw}} \text {. }
$$

\subsection{2 锅炉过热器倁耗散率}

当忽略保热系数 $\varphi$ 的影响时，第 $i$ 流程烟气放热过 程的䛈耗散率 $\dot{E}_{\phi, \mathrm{fg}, i}$ 可表示为

$$
\dot{E}_{\phi, \mathrm{fg}, i}=0.5 \dot{m}_{\mathrm{fg}} \bar{c}_{\mathrm{p}, \mathrm{sht}, i}\left(T_{\mathrm{fg}, i}^{2}-T_{\mathrm{fg}, i^{\prime}}^{2}\right) .
$$

管内蒸汽吸热过程的积耗散率 $\dot{E}_{\phi, \mathrm{st}}$ 可表示为

$$
\dot{E}_{\phi, \mathrm{st}}=\dot{m}_{\mathrm{st}}\left(c_{\mathrm{p}, 3}+c_{\mathrm{p}, 4}\right) / 2 \cdot\left(T_{3}^{2}-T_{4}^{2}\right) / 2 .
$$

忽略烟气流动过程所引起的䍉耗散率，过热器总 的炇耗散率 $\dot{E}_{\phi, \mathrm{sht}}$ 为

$$
\dot{E}_{\phi, \mathrm{sht}}=\sum_{i=1}^{3} \dot{E}_{\phi, \mathrm{fg}, i}+\dot{E}_{\phi, \mathrm{st}} .
$$




\subsection{3 锅炉经济器炽耗散率}

当忽略保热系数 $\varphi$ 的影响时, 烟气放热过程的䍉耗 散率 $\dot{E}_{\phi, \mathrm{fg}}$ 可表示为

$$
\dot{E}_{\phi, \mathrm{fg}}=0.5 \dot{m}_{\mathrm{fg}} \bar{c}_{\mathrm{p}, \mathrm{eco}}\left(T_{5}^{2}-T_{6}^{2}\right) .
$$

管内水吸热过程的䍉耗散率 $\dot{E}_{\phi, \mathrm{wt}}$ 表示为

$$
\dot{E}_{\phi, \mathrm{wt}}=\dot{m}_{\mathrm{wt}}\left(\bar{c}_{\mathrm{p}, 1}+\bar{c}_{\mathrm{p}, 2}\right) / 2 \cdot\left(T_{1}^{2}-T_{2}^{2}\right) / 2 \text {. }
$$

忽略烟气流动过程所引起的䍉耗散率, 经济器总 的䛈耗散率 $\dot{E}_{\phi, \mathrm{eco}}$ 可表示为

$$
\dot{E}_{\phi, \text { eco }}=\dot{E}_{\phi, \mathrm{fg}}+\dot{E}_{\phi, \mathrm{wt}} .
$$

\subsection{4 整体锅炉总䍉耗散率}

锅炉总的䛈耗散率 $\dot{E}_{\phi, \mathrm{sb}}$ 为蒸发器、过热器和经济 器的沵耗散率之和, 所以有

$\dot{E}_{\phi, \mathrm{sb}}=\dot{E}_{\phi, \text { evap }}+\dot{E}_{\phi, \mathrm{sht}}+\dot{E}_{\phi, \mathrm{eco}}$.

\section{3 整体锅炉耗功率}

在锅炉蒸发器中，管外烟气和管内工质均需保持 一定的流速来进行正常的对流换热. 由于整体锅炉计 算中烟气耗功率大多由浴轮增压机组中的废气涡轮提 供, 不需要额外消耗附加能量. 因此, 烟气耗功率不计 入总耗功率, 仅将工质侧的耗功率计入. 本文主要考虑 锅炉换热设备部分的性能, 不考虑其他辅机的性能.

\subsection{1 锅炉蒸发器耗功率}

由于本文研究的锅炉属于自然循环锅炉, 蒸发器 管内工质的流动由工质温差造成的密度差提供循环驱 动力, 工质的流动不需要消耗额外的洜功率. 因此, 蒸 发器的总耗功率 $P_{\mathrm{evap}}$ 为

$P_{\text {evap }}=0$.

\subsection{2 锅炉过热器耗功率}

对于锅炉过热器, 第 $i$ 流程蒸汽流动耗功率 $P_{\mathrm{st}, i}$ 为

$P_{\mathrm{st}, i}=\dot{m}_{\mathrm{st}} \Delta p_{\mathrm{st}, i} /\left(\eta_{\mathrm{p}} \rho_{\mathrm{st}, i}\right)$,

式中, $\Delta p_{\mathrm{st}, i}$ 为蒸汽流经第 $i$ 流程管束时的压降, $\eta_{\mathrm{p}}$ 为给水
葲效率(厡的有效功率与轴功率之比), $\rho_{\mathrm{st}, i}$ 为第 $i$ 流程蒸 汽的平均密度.

由此可得锅炉过热器总耗功率 $P_{\text {sht }}$ 为

$P_{\mathrm{sht}}=\sum_{i=1}^{3} P_{\mathrm{st}, i}$

\subsection{3 锅炉经济器耗功率}

类似于锅炉过热器, 锅炉经济器总耗功率 $P_{\mathrm{eco}}$ 为

$P_{\text {eco }}=\dot{m}_{\mathrm{wt}} \Delta p_{\mathrm{wt}} /\left(\eta_{\mathrm{p}} \rho_{\mathrm{wt}}\right)$,

式中, $\Delta p_{\mathrm{wt}}$ 为水流经经济器管束时的压降, $\eta_{\mathrm{p}}$ 为百效率, $\rho_{\mathrm{wt}}$ 为经济器中水的平均密度.

\subsection{4 整体锅炉总耗功率}

整体锅炉耗功率 $P_{\mathrm{sb}}$ 为蒸发器、过热器和经济器 三者耗功率之和, 即

$P_{\mathrm{sb}}=P_{\text {evap }}+P_{\text {sht }}+P_{\text {eco }}$.

\section{3 整体锅炉熕耗散率和耗功率线性加权和 最小构形优化}

\section{1 优化问题描述和求解流程}

单独优化锅炉耗功率 $P_{\mathrm{sb}}$ 或沵耗散率 $E_{\mathrm{g}, \mathrm{sb}}$ 不能充分 反映其综合性能. 因此, 为了更好地满足实际需要, 本 文首先采用线性加权法 ${ }^{[12 ~ 16,37,38,40 \sim 43]}$ 建立䛈耗散率和 耗功率组成的复合函数作为锅炉性能优化指标:

$F_{\mathrm{EP}}=a_{0} \widetilde{E}_{\phi, \mathrm{sb}}+\left(1-a_{0}\right) \widetilde{P_{\mathrm{sb}}}$,

式中, $a_{0}$ 为复合函数加权系数, $\widetilde{E}_{\phi, \mathrm{sb}}\left(=\dot{E}_{\phi, \mathrm{sb}} / \dot{E}_{\phi, \mathrm{sb}, \mathrm{int}}\right)$ 和 $\widetilde{P_{\mathrm{sb}}}\left(=P_{\mathrm{sb}} / P_{\mathrm{sb}, \text { int }}\right)$ 分别为整体锅炉的无量纲炇耗散率和 无量纲耗功率, $\dot{E}_{\phi, \mathrm{sb}, \mathrm{int}}$ 和 $P_{\mathrm{sb}, \text {,int }}$ 分别为初始䍉耗散率和初 始耗功率; 由于 $\widetilde{E}_{\phi, \mathrm{sb}}$ 比 $\widetilde{P}_{\mathrm{sb}}$ 小得多, $a_{0}$ 需取较大的值才能 使得加权后的 $\widetilde{E}_{\phi, \mathrm{sb}}$ 和 $\widetilde{P}_{\mathrm{sb}}$ 大小相当. 炇耗散率越小越有 利于提高锅炉的整体传热性能，降低传热过程的不可 逆性; 耗功率越小越有利于减少洜功投入, 提高锅炉 的经济性. 两者的加权和复合函数 $F_{\mathrm{EP}}$ 将使得锅炉的传 热过程不可逆性和耗功率得到折中, 均不至于过大, 复 合函数越小对应的锅炉综合性能越优. 
在锅炉总换热率和换热面积一定的约束条件下, 本文以第III对流蒸发管束无量纲管外径 $\widetilde{d}_{3 \mathrm{cet}}$ 、过热器 无量纲管外径 $\tilde{d}_{\mathrm{sht}}$ 和经济器无量纲管外径 $\tilde{d}_{\mathrm{eco}}$ 为优化变 量，以复合函数 $F_{\mathrm{EP}}$ 为优化目标，对整体锅炉开展构形 优化. 在优化结果中, 下标“m”, “mm”和“mmm”分别表 示复合函数的一次、二次和三次最小化，下标“opt”, “oo”和“ooo”分别表示优化变量的一次、二次和三次 最佳. 表1给出了优化过程中部分变量的初始值及变化 范围, 优化变量 $\widetilde{d}_{3 \mathrm{cet}}, \widetilde{d}_{\mathrm{sht}}$ 和 $\widetilde{d}_{\mathrm{eco}}$ 的变化范围由锅炉实际 尺寸确定.

\section{2 䍉耗散率和耗功率复合优化}

图3 5分别给出了保热系数 $\varphi$ 对复合函数 $F_{\mathrm{EP}}$ 与 $\widetilde{d}_{3 \mathrm{cet}}$ 关系、 $F_{\mathrm{EP}}$ 与 $\widetilde{d}_{\mathrm{sht}}$ 关系和 $F_{\mathrm{EP}}$ 与 $\widetilde{d}_{\mathrm{eco}}$ 关系的影响. 由图 可知，不同 $\varphi$ 下，随着 $\widetilde{d}_{3 \mathrm{cet}}$ 和 $\widetilde{d}_{\mathrm{eco}}$ 的增加，锅炉的䛈耗散 率和耗功率均减小, 最终使得 $F_{\mathrm{EP}}$ 均逐渐减小. 随着 $\tilde{d}_{\mathrm{sht}}$ 的增加, 锅炉的椥耗散率略有减小, 过热蒸汽产量 $\dot{m}_{\mathrm{st}}$
增大, 过热器管束内压降 $\Delta p_{\mathrm{st}}$ 减小但幅度越来越小; 这 使得 $\widetilde{d}_{\mathrm{sh}}$ 较小时 $\dot{m}_{\mathrm{st}}$ 增大的幅度小于 $\Delta p_{\mathrm{st}}$ 减小的幅度, 耗 功率先减小; $\widetilde{d}_{\mathrm{sht}}$ 较大时 $\dot{m}_{\mathrm{st}}$ 增大的幅度大于 $\Delta p_{\mathrm{st}}$ 减小的 幅度, 耗功率后增大; 由于耗功率的变化幅度均大于䍉 耗散率的变化幅度, 最终导致 $F_{\mathrm{EP}}$ 先减小后增大. 当保 热系数 $\varphi=0.98$ 时, 存在最佳无量纲过热器管外 径 $\widetilde{d}_{\mathrm{sht}, \mathrm{opt}}=1.17$ 使得 $F_{\mathrm{EP}}$ 取得极小值 0.981 . 与初始设计 点相比, 优化后的锅炉整体复合函数 $F_{\mathrm{EP}}$ 、炇耗散率 $\dot{E}_{\mathrm{sb}}$ 和耗功率 $P_{\mathrm{sp}}$ 分别减小了 $1.9 \%, 1.12 \%$ 和 $4.26 \%$. 综合分 析可得，优化后的整体锅炉在各复合函数中的两个性 能指标所表征的性能均得到改善, 即整体锅炉优化后, 综合性能的提高是相应两个性能均得到提升的综合结 果. 在 $\widetilde{d}_{3 \mathrm{cet}}$ 和 $\widetilde{d}_{\mathrm{eco}}$ 的讨论范围内, $F_{\mathrm{EP}}-\widetilde{d}_{3 \mathrm{cet}}$ 和 $F_{\mathrm{EP}}-\widetilde{d}_{\mathrm{eco}}$ 曲 线均不存在极小值点. 实际设计中, 当 $\varphi=0.98$ 时, 应选 取最佳设计值 $\widetilde{d}_{3 \mathrm{cet}}=1.2$ 和 $\widetilde{d}_{\mathrm{eco}}=1.1$ 使得 $F_{\mathrm{EP}}$ 取得讨论 范围内的最小值 0.758 和 0.958 . 由图4还可以看出, 随着 保热系数 $\varphi$ 的增加, $F_{\mathrm{EP}}$ 减小, 锅炉整体综合性能得到提

表 1 部分变量初始值及变化范围

Table 1 Initial values and variation ranges of some parameters

\begin{tabular}{|c|c|c|c|c|}
\hline 参数 & 符号 & 初始值 & 变化范围 & 单位 \\
\hline 锅炉保热系数 & $\varphi$ & 0.98 & $0.9 \sim 0.98$ & - \\
\hline 过余空气系数 & $\alpha$ & 1.2 & $1.18 \sim 1.38$ & - \\
\hline 加权系数 & $a_{0}$ & 0.9 & $0 \sim 1$ & - \\
\hline 第I对流蒸发管束管外径 & $d_{1 \mathrm{cet}}$ & 38 & - & $\mathrm{mm}$ \\
\hline 第I对流蒸发管束管排数 & $z_{\text {lcet }}$ & 1 & - & - \\
\hline 第I对流蒸发管束单排管数 & $n_{1 \mathrm{cet}}$ & 37 & - & - \\
\hline 第II对流蒸发管束管外径 & $d_{2 \mathrm{cet}}$ & 30 & - & $\mathrm{mm}$ \\
\hline 第II对流蒸发管束管排数 & $z_{2 \text { cet }}$ & 2 & - & - \\
\hline 第II对流蒸发管束单排管数 & $n_{2 \text { cet }}$ & 37 & - & - \\
\hline 第III对流蒸发管束管外径 & $d_{3 \mathrm{cet}}$ & 30 & $24 \sim 36$ & $\mathrm{~mm}$ \\
\hline 第III对流蒸发管束管排数 & $z_{3 \mathrm{cet}}$ & 6 & - & - \\
\hline 第III对流蒸发管束单排管数 & $n_{3 \mathrm{cet}}$ & 74 & - & - \\
\hline 过热器管束管外径 & $d_{\text {sht }}$ & 20 & $16 \sim 26$ & $\mathrm{~mm}$ \\
\hline 过热器管束管排数 & $z_{\text {sht }}$ & 11 & - & - \\
\hline 过热器管束单排管数 & $n_{\text {sht }}$ & 78 & - & - \\
\hline 蛇形管束管外径 & $d_{\mathrm{eco}}$ & 25 & $20 \sim 28$ & $\mathrm{~mm}$ \\
\hline 蛇形管束管排数 & $z_{\text {eco }}$ & 10 & - & - \\
\hline 蛇形管束管组数 & $n_{\mathrm{eco}}$ & 17 & - & - \\
\hline
\end{tabular}




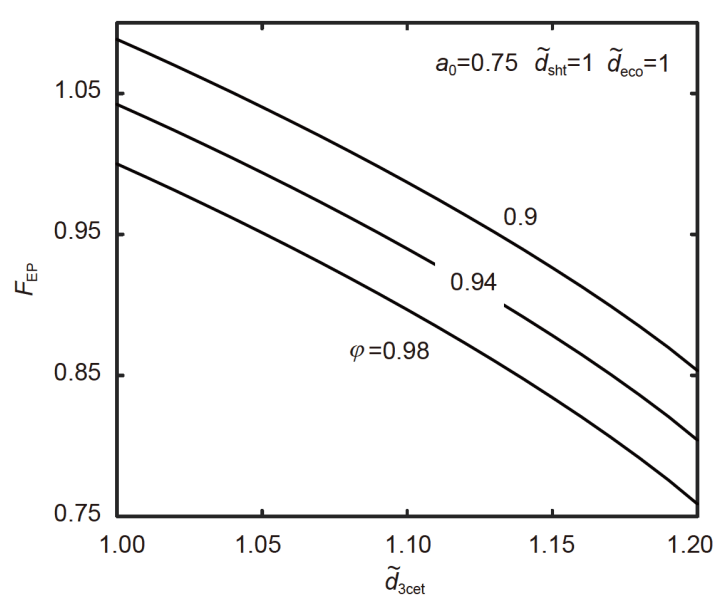

图 $3 \varphi$ 对 $F_{\mathrm{EP}}$ 与 $\tilde{d}_{3 \mathrm{cet}}$ 关系的影响

Figure 3 Effect of $\varphi$ on the relationship of $F_{\mathrm{EP}}$ versus $\tilde{d}_{3 \mathrm{cet}}$.

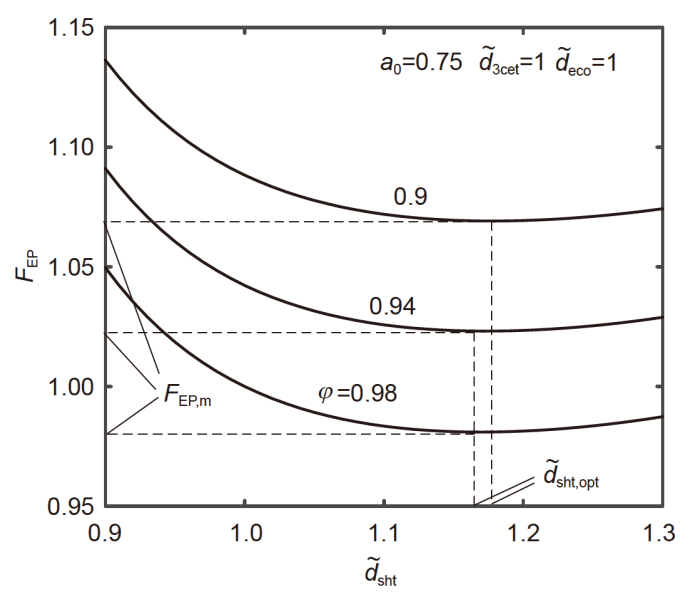

图 $4 \varphi$ 对 $F_{\mathrm{EP}}$ 与 $\tilde{d}_{\mathrm{sh}}$ 关系的影响

Figure 4 Effect of $\varphi$ on the relationship of $F_{\mathrm{EP}}$ versus $\widetilde{d}_{\text {sht }}$.

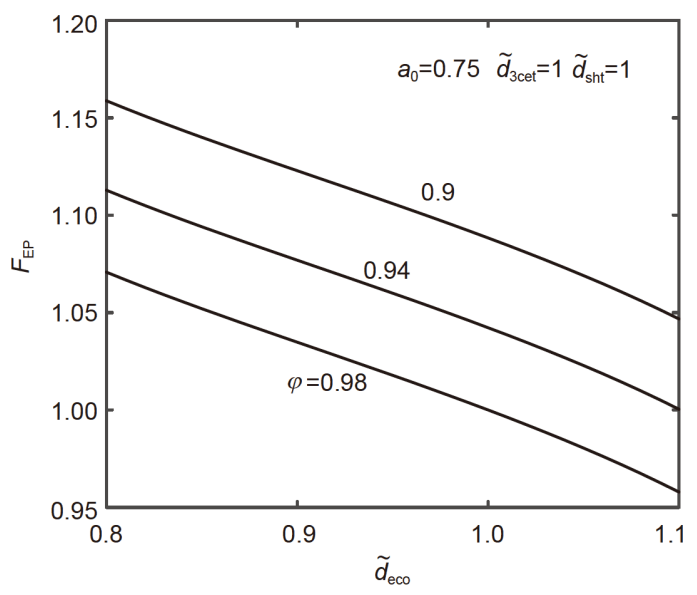

图 $5 \varphi$ 对 $F_{\mathrm{EP}}$ 与 $\tilde{d}_{\mathrm{eco}}$ 关系的影响

Figure 5 Effect of $\varphi$ on the relationship of $F_{\mathrm{EP}}$ versus $\widetilde{d}_{\text {eco. }}$.
升. 这是由于保热系数的增加致使锅炉䛈耗散率减小, 耗功率增大, 由于积耗散率所占权重较大, 故复合函 数 $F_{\mathrm{EP}}$ 减小.

图6给出了复合函数加权系数 $a_{0}$ 对 $F_{\mathrm{EP}}$ 与 $\widetilde{d}_{\mathrm{sht}}$ 关系 的影响. 由图可知, 当 $a_{0}$ 分别取 $0,0.25,0.5$ 和 0.75 时, 无 量纲管外径 $\widetilde{d}_{\mathrm{sht}}$ 分别存在最佳值1.12, 1.12, 1.13和 1.17 , 使得 $F_{\mathrm{EP}}$ 取得最小值 $0.952,0.963,0.974$ 和 0.981 ; 当 $a_{0}$ 取 1 时, 随着 $\tilde{d}_{\mathrm{sht}}$ 的增大, $F_{\mathrm{EP}}$ 近似线性减小. 在不同 $a_{0}$ 下均 存在 $\widetilde{d}_{\mathrm{sht}, \mathrm{opt}}, F_{\mathrm{EP}, \mathrm{m}}$ 随 $a_{0}$ 的增大而增大.

图7给出了一次最小复合函数 $F_{\mathrm{EP}, \mathrm{m}}$ 和一次最佳无 量纲管外径 $\tilde{d}_{\text {sht,opt }}$ 与 $\widetilde{d}_{3 \mathrm{cet}}$ 的关系. 由图可知, 在 $\widetilde{d}_{3 \mathrm{cet}}$ 的讨

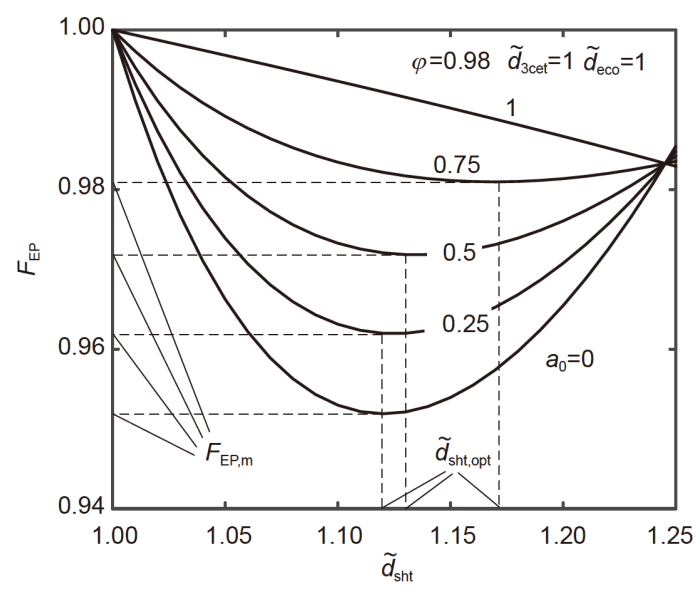

图 $6 a_{0}$ 对 $F_{\mathrm{EP}}$ 与 $\tilde{d}_{\mathrm{sht}}$ 关系的影响

Figure 6 Effect of $a_{0}$ on the relationship of $F_{\mathrm{EP}}$ versus $\tilde{d}_{\text {sht }}$.

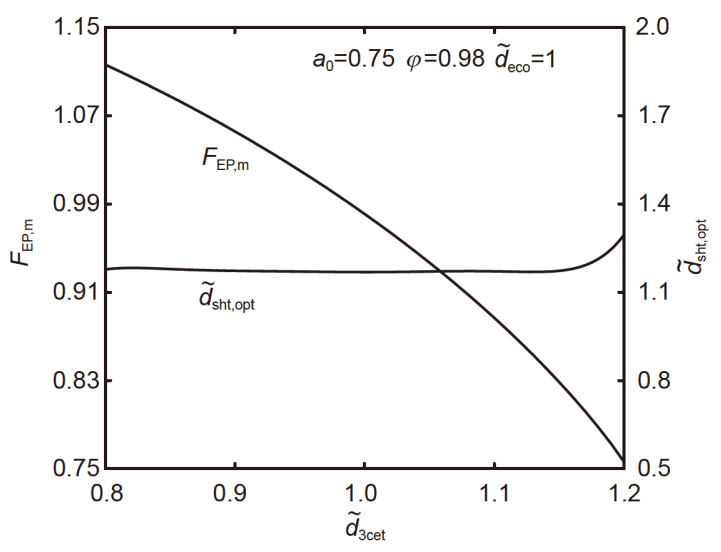

图 $7 F_{\mathrm{EP}, \mathrm{m}}$ 和 $\tilde{d}_{\mathrm{sht}, \mathrm{opt}}$ 与 $\tilde{d}_{3 \mathrm{cet}}$ 的关系

Figure 7 Relationships of $F_{\mathrm{EP}, \mathrm{m}}$ and $\tilde{d}_{\text {sht,opt }}$ versus $\tilde{d}_{3 \mathrm{cet}}$. 
论范围内, $F_{\mathrm{EP}, \mathrm{m}}-\widetilde{d}_{3 \mathrm{cet}}$ 曲线不存在极值点. 因此, 实际设 计中应选择最佳设计值 $\widetilde{d}_{3 \mathrm{cet}}=1.2, \quad \widetilde{d}_{\mathrm{sht}, \mathrm{oo}}=1.3$ 和 $F_{\mathrm{EP}, \mathrm{mm}}=0.756$ 使得锅炉综合性能趋于最优. 与初始设 计点相比, 二次优化后的锅炉复合函数 $F_{\mathrm{EP}}$ 、䍉耗散 率 $\dot{E}_{\phi, \mathrm{s} b}$ 和耗功率 $P_{\mathrm{sb}}$ 分别降低了 $24.4 \%, 4.98 \%$ 和 $82.51 \%$. 进一步分析, 当进行三变量 $\widetilde{d}_{3 \mathrm{cet}}, \widetilde{d}_{\mathrm{sht}}$ 和 $\widetilde{d}_{\mathrm{eco}}$ 优化时, 取 $\widetilde{d}_{\mathrm{eco}, \mathrm{opt}}=1.1, \widetilde{d}_{3 \mathrm{cet}, \mathrm{oo}}=1.2$ 和 $\widetilde{d}_{\mathrm{sht}, \mathrm{ooo}}=1.3$ 可使得 $F_{\mathrm{EP}}$ 取得 三次最小值 $F_{\mathrm{EP}, \mathrm{mmm}}=0.737$. 与初始设计点相比, 三次 优化后整体锅炉的 $F_{\mathrm{EP}}, \dot{E}_{\phi, \mathrm{sb}}$ 和 $P_{\mathrm{sb}}$ 分别减小了 $26.3 \%$, $6.56 \%$ 和 $85.17 \%$. 综合分析可得，复合函数下，整体锅 炉的最优性能形成机理是不随优化自由度的增加而改 变的, 且其综合性能随优化自由度的增加而不断提高.

图 8 给出了无量纲换热率 $\widetilde{Q}_{\mathrm{sb}}$ 对 $F_{\mathrm{EP}, \mathrm{mmm}}, \widetilde{d}_{\mathrm{sht}, 0 \mathrm{ooo}}$, $\widetilde{d}_{3 \mathrm{cet}, \mathrm{oo}}$ 和 $\widetilde{d}_{\mathrm{eco}, \mathrm{opt}}$ 的影响. 由图可知, 随着 $\widetilde{Q}_{\mathrm{sb}}$ 的增大, $F_{\mathrm{EP}, \mathrm{mmm}}$ 逐渐增大, $\widetilde{d}_{\mathrm{sht}, \mathrm{ooo}}, \widetilde{d}_{3 \mathrm{cet}, \mathrm{oo}}$ 和 $\widetilde{d}_{\mathrm{eco}, \mathrm{opp}}$ 均保持不变. 增大 $\widetilde{Q}_{\mathrm{sb}}$ 会降低目标 $F_{\mathrm{EP}}$ 下整体锅炉的综合性能，但不 影响其最优构形。随着换热率的增加，锅炉炇耗散率 和耗功率均增大，故复合函数 $F_{\mathrm{EP}}$ 增大. 换热率对整体 锅炉最优构形不产生影响.

\section{4 基于NSGA-II算法的整体锅炉两目标构 形优化}

上节对整体锅炉的优化研究采用了线性加权法将 多目标转化为单目标进行研究. 在工程应用中, 设计需

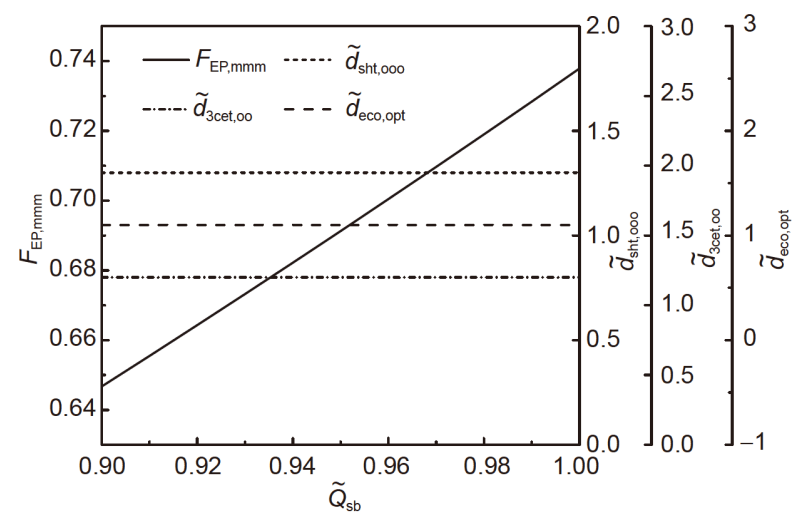

图 $8 \widetilde{Q}_{\mathrm{sb}}$ 对 $F_{\mathrm{EP}, \mathrm{mmm}}, \widetilde{d}_{\mathrm{sht}, \mathrm{ooo}}, \widetilde{d}_{3 \mathrm{cet}, \mathrm{oo}}$ 和 $\widetilde{d}_{\mathrm{eco}, \mathrm{opt}}$ 的影响 Figure 8 Effect of $\widetilde{Q}_{\mathrm{sb}}$ on $F_{\mathrm{EP}, \mathrm{mmm}}, \widetilde{d}_{\text {sht,ooo }}, \widetilde{d}_{3 \text { cet,oo }}$ and $\widetilde{d}_{\text {eco,opt }}$.
求不一定是由两个目标函数进行线性加权得到的, 且 设计人员们往往追求研究对象在多个目标下的性能提 升. 非劣解排序遗传算法(NSGA-II) 是一种工程上广泛 采用的多目标优化算法, 可用来解决相互矛盾的多个 目标的优化问题 ${ }^{[4-55]}$. 图9给出了NSGA-II算法流程 图, 多目标优化算法给出的是一系列非劣解, 相对应 的目标函数值称为Pareto前沿.

图10给出了基于无量纲䛈耗散率 $\widetilde{E}_{\phi, \mathrm{s}}$ 最小目标和 无量纲耗功率 $\widetilde{P}_{\mathrm{sb}}$ 最小目标的Pareto前沿, Pareto前沿的 每个点均为锅炉在特定优化目标下的锅炉性能最优解 集. 理想点 $\left(\dot{E}_{\phi, \mathrm{sb}}\right.$ 最小且 $P_{\mathrm{sb}}$ 最小 $)$ 和非理想点 $\left(\dot{E}_{\phi, \mathrm{s}}\right.$ 最大 且 $P_{\mathrm{sb}}$ 最大)均不能实现; $\mathrm{A}$ 点可看作单独优化 $\widetilde{P_{\mathrm{sb}}}$ 时, 最 小 $\widetilde{P}_{\mathrm{sb}}$ 对应的最优解; $\mathrm{B}$ 点可看作单独优化 $\widetilde{E}_{\phi, \mathrm{sb}}$ 时, 最 小 $\widetilde{E}_{\phi, \mathrm{sb}}$ 对应的最优解. 图11给出了Pareto最优解集所对 应的锅炉最优构形 $\left(\widetilde{d}_{\mathrm{sht}, \mathrm{ooo}}, \widetilde{d}_{3 \mathrm{cet}, \mathrm{oo}}\right.$ 和 $\left.\widetilde{d}_{\mathrm{eco}, \mathrm{opt}}\right)$. 由图可知, 不同Pareto 最优解所对应的 $\widetilde{d}_{\mathrm{sht}, \mathrm{ooo}}$ 不同, 但 $\widetilde{d}_{3 \mathrm{cet}, \mathrm{oo}}$

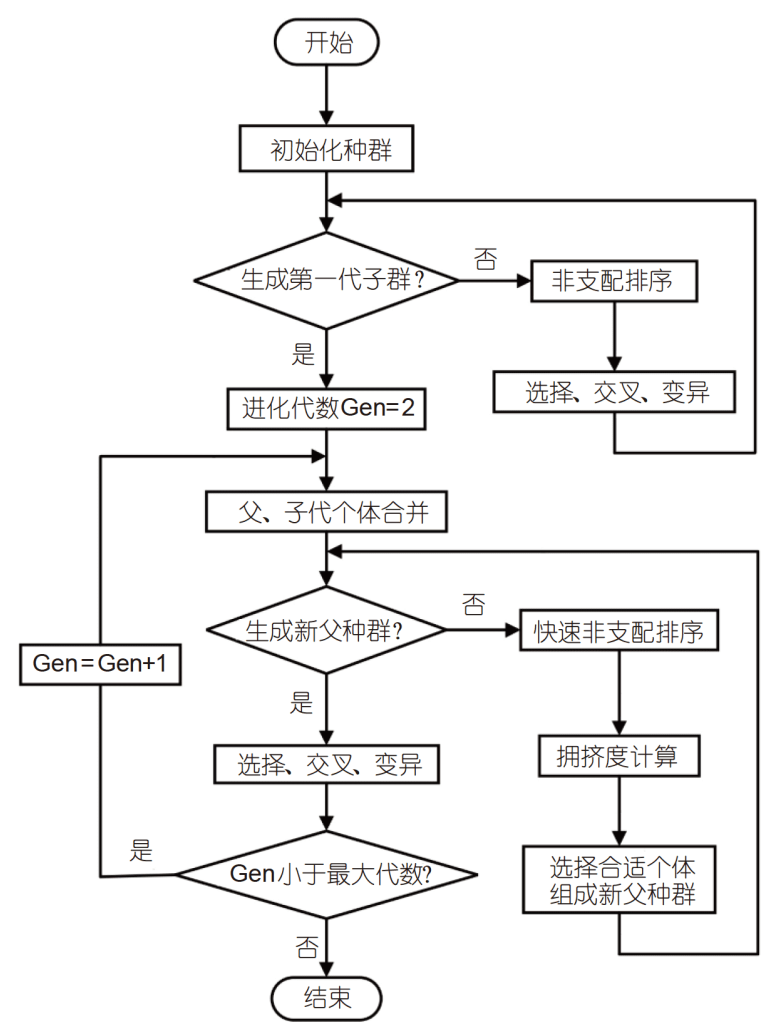

图 9 NSGA-II算法流程图

Figure 9 Program flow chart for NSGA-II algorithm. 


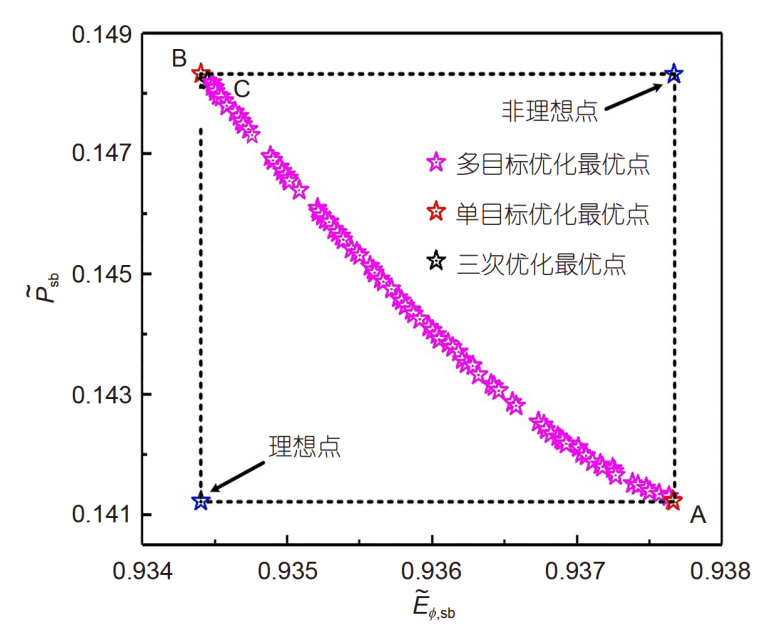

图 10 (网络版彩图)锅炉两目标优化Pareto前沿

Figure 10 (Color online) Pareto front of the boiler based on biobjective optimization.

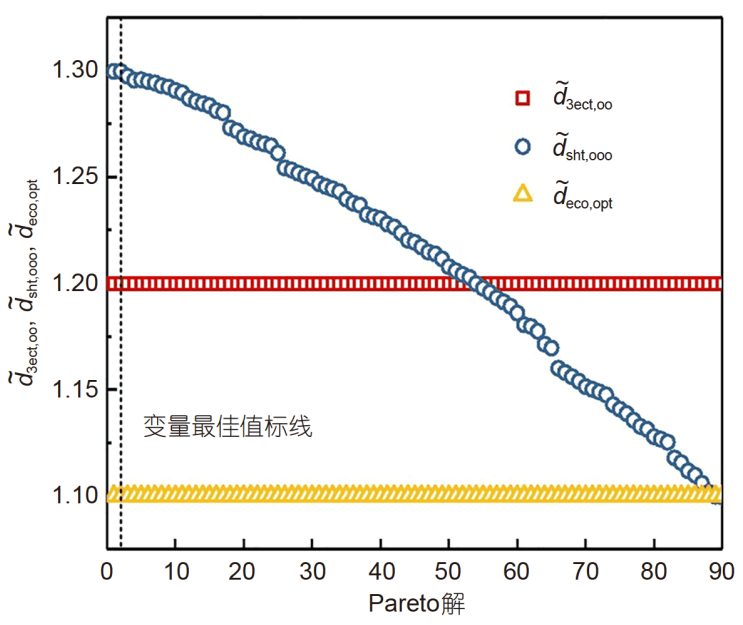

图 11 (网络版彩图)Pareto解集对应的锅炉最优构形

Figure 11 (Color online) Optimal constructs of the boiler corresponding to Pareto optimal set.

和 $\widetilde{d}_{\mathrm{eco}, \mathrm{opt}}$ 保持不变. 同样地, 图10中Pareto前沿包含了 线性加权法基于目标函数 $\widetilde{E}_{\phi, \mathrm{sb}}$ 和 $\widetilde{P}_{\mathrm{sb}}$ 的三次最优解(点 C), 相应的三个变量最佳值在图11中标出. 图10和11的 优化结果可为考虑炇耗散率和耗功率的锅炉多目标性 能优化与结构设计提供更广泛的选择方案.

\section{5 结论}

本文建立了整体锅炉模型，在总换热面积一定的 约束条件下，以第III对流蒸发管束无量纲管外 径 $\widetilde{d}_{3 \mathrm{cet}}$ 、过热器无量纲管外径 $\tilde{d}_{\mathrm{sht}}$ 和经济器无量纲管 外径 $\widetilde{d}_{\mathrm{eco}}$ 为优化变量, 首先以䛈耗散率和耗功率组成的 复合函数 $F_{\mathrm{EP}}$ 为优化目标, 得到了整体锅炉的最优构形 及最优性能, 并分析了初始设计参数对优化结果的影 响; 然后用NSGA-II算法进行了整体锅炉的两目标优 化, 给出了其相应的Pareto前沿及锅炉最优构形. 研究 结论如下.

(1) 单变量优化中, 当保热系数 $\varphi=0.98$ 时, 过热器 无量纲管外径 $\widetilde{d}_{\mathrm{sht}}$ 在最佳值1.17使得复合函数 $F_{\mathrm{EP}}$ 取得 最小值0.981. 三变量优化中, $\tilde{d}_{\mathrm{sht}}$ 存在三次最佳值 1.3 使 得复合函数 $F_{\mathrm{EP}}$ 取得三次最小值 0.737 . 与初始设计点相 比，优化后整体锅炉的复合函数 $F_{\mathrm{EP}}$ 最大降低了 $26.3 \%$. 由于变量 $\widetilde{d}_{3 \mathrm{cet}}$ 和 $\widetilde{d}_{\mathrm{eco}}$ 均在所讨论范围的最大边界处取 得最佳值, 故各个优化目标下的最佳对流蒸发管束外 径 $\tilde{d}_{3 \mathrm{cet}, 0 \mathrm{o}}$ 和最佳经济器管外径 $\tilde{d}_{\mathrm{eco}, \mathrm{opp}}$ 始终不变, 其值分 别为 1.2 和 1.1 . 随着优化自由度的增加, 整体锅炉的综 合性能不断提高.

（2）复合函数加权系数 $a_{0}$ 同样会对整体锅炉的优 化结果产生定性和定量影响. 整体锅炉 $F_{\mathrm{EP}, \mathrm{m}}$ 随 $\varphi$ 的增大 而减小. 整体锅炉综合性能随过余空气系数 $\alpha$ 的增大而 降低, 随燃油消耗率 $B$ 的增大而提高. 在目标 $F_{\mathrm{EP}}$ 下, 换 热率的提高会降低锅炉的综合性能.

(3) 在两目标优化中，整体锅炉基于目标函数 $F_{\mathrm{EP}}$ 所建立Pareto前沿中的理想点和非理想点均不能实现, 且Pareto前沿包含了单目标优化最优解和基于线性加 权法的最优解, 相应的最优构形可为锅炉多目标性能 优化与结构设计提供更广泛的选择方案, 从而更好地 指导工程实践.

(4) 烍耗散率为船用锅炉整体设计提供了新的优 化目标，将䛈理论与构形理论相结合所得结果可为实 际锅炉的优化设计提供新的理论指导. 


\section{参考文献}

1 Bejan A. Shape and Structure, from Engineering to Nature. Cambridge: Cambridge University Press, 2000

2 Bejan A, Lorente S. Design with Constructal Theory. New Jersey: Wiley, 2008

3 Chen L G. Progress in study on constructal theory and its applications. Sci China Tech Sci, 2012, 55: 802-820

4 Feng H J, Chen L G, Xie Z H. Multi-disciplinary, multi-objective and multi-scale constructal optimizations for heat and mass transfer processes performed in Naval University of Engineering, a review. Int J Heat Mass Transfer, 2017, 115: 86-98

5 Chen L G, Feng H J, Xie Z H, et al. Progress of constructal theory in China over the past decade. Int J Heat Mass Transfer, 2019, 130: 393-419

6 Lorente S, Bejan A. Current trends in constructal law and evolutionary design. Heat Trans Asian Res, 2019, 48: 3574-3589

7 Chen L G, Yang A B, Feng H J, et al. Constructal design progress for eight types of heat sinks. Sci China Tech Sci, 2020, 63: 879-911

8 Yu M J, Lai X T, Xiao H, et al. A study on flow and heat transfer characteristics of a constructal bifurcation filler in the circular tube. Appl Thermal Eng, 2021, 183: 116205

9 Yu M J, Xin F, Lai X T, et al. Study of oscillating flows through a novel constructal bifurcation Stirling regenerator. Appl Thermal Eng, 2021, 184: 116413

10 Chen L G, Wu W J, Feng H J. Constructal Design for Heat Conduction. London: Book Publisher International, 2021

11 Chen L G, Feng H J, Wu Z X, et al. Progress in theoretical study on constructal optimizations of marine steam power plants (in Chinese). Sci Sin Tech, 2021, doi: 10.1360/SST-2020-0393 [陈林根, 冯辉君, 吴志祥, 等. 船海用蒸汽动力装置构形优化理论研究进展. 中国科学: 技术科学, 2021, doi: 10.1360/SST-2020-0393]

12 Xie Z J, Feng H J, Chen L G, et al. Constructal design for supercharged boiler evaporator. Int J Heat Mass Transfer, 2019, 138: 571-579

13 Feng H, Xie Z J, Chen L G, et al. Constructal design for supercharged boiler superheater. Energy, 2020, 191: 116484

14 Tang W, Feng H J, Chen L G, et al. Constructal design for a boiler economizer. Energy, 2021, 223: 120013

15 Gulotta T M, Guarino F, Cellura M, et al. Constructal law optimization of a boiler. Int J Heat Technol, 2017, 35: 297-305

16 Gulotta T M, Guarino F, Cellura M, et al. A constructal law optimization of a boiler inspired by life cycle thinking. Thermal Sci Eng Prog, 2018, 6: $380-387$

17 Guo Z Y, Zhu H Y, Liang X G. Entransy-A physical quantity describing heat transfer ability. Int J Heat Mass Transfer, 2007, 50: 2545-2556

18 Li Z X, Guo Z Y. Field Synergy Principle of Heat Convection Optimization (in Chinese). Beijing: Science Press, 2010 [李志信, 过增元. 对流传 热优化的场协同理论. 北京: 科学出版社, 2010]

19 Liu W, Liu Z C, Jia H, et al. Entransy expression of the second law of thermodynamics and its application to optimization in heat transfer process. Int J Heat Mass Transfer, 2011, 54: 3049-3059

20 Chen L G. Progress in entransy theory and its applications. Chin Sci Bull, 2012, 57: 4404-4426

21 Chen Q, Liang X G, Guo Z Y. Entransy theory for the optimization of heat transfer-A review and update. Int J Heat Mass Transfer, 2013, 63: $65-81$

22 Chen L G. Progress in optimization of mass transfer processes based on mass entransy dissipation extremum principle. Sci China Tech Sci, 2014, 57: $2305-2327$

23 Cheng X T, Zhao J M, Liang X G. Discussion on the extensions of the entransy theory. Sci China Technol Sci, 2017, 60: 363-373

24 Chen L G, Xiao Q H, Feng H J. Constructal optimizations for heat and mass transfers based on the entransy dissipation extremum principle, performed at the Naval University of Engineering: A review. Entropy, 2018, 20: 74

25 Chen X, Zhao T, Zhang M Q, et al. Entropy and entransy in convective heat transfer optimization: A review and perspective. Int J Heat Mass Transfer, 2019, 137: 1191-1220

26 Liang X G, Chen Q, Guo Z Y. Entransy Theory for Heat Transfer and its Applications (in Chinese). Beijing: Science Press, 2019 [梁新刚, 陈群, 过增元. 传热烦理论及其应用. 北京: 科学出版社, 2019]

27 Wang W H, Cheng X T, Liang X G. Entropy and entransy analyses and optimizations of the rankine cycle. Energy Convers Manage, 2013, 68: $82-88$

28 Cheng X T, Liang X G. Entransy variation associated with work. Int J Heat Mass Transfer, 2015, 81: 167-170 
61: 1877-1888 [孟继安, 李志信. 管束布置对凝汽器性能影响的䍉分析及其应用. 科学通报, 2016, 61: 1877-1888]

30 Wang X J, He Y L, Wang Z D, et al. Microscopic expression of entransy in ideal gas system for diatomic molecules. Int J Heat Mass Transfer, 2018, 127: 1347-1350

31 Feng H J, Chen L G, Xie Z H. Constructal entransy dissipation rate minimization for X-shaped vascular networks. Sci China Tech Sci, 2019, 62: 2195-2203

32 Cheng X T, Liang X G. Entransy functions for steady heat transfer. Sci China Tech Sci, 2019, 62: 1726-1734

33 Zhao T, Liu D, Chen Q. A collaborative optimization method for heat transfer systems based on the heat current method and entransy dissipation extremum principle. Appl Thermal Eng, 2019, 146: 635-647

34 Li K, Wen J, Liu Y, et al. Application of entransy theory on structure optimization of serrated fin in plate-fin heat exchanger. Appl Thermal Eng, 2020, 173: 114809

35 Kim K H, Jung Y G, Han C H. Energy and entransy characteristic analysis of heat exchangers depending on heat exchanger type. Trans Korean Hydrogen New Energy Society, 2020, 31: 112-121

36 Wang S, Zhang W, Feng Y Q, et al. Entropy, entransy and exergy analysis of a dual-loop organic Rankine cycle (DORC) using mixture working fluids for engine waste heat recovery. Energies, 2020, 13: 1301

37 Feng H J, Chen L G, Wu Z X, et al. Constructal optimization for an organic fluid shell-and-tube heat exchanger based on entransy theory (in Chinese). Sci Sin Tech, 2020, 50: 1577-1587 [冯辉君, 陈林根, 吴志祥, 等. 基于积理论的有机工质管壳式换热器构形优化. 中国科学: 技术 科学, 2020, 50: 1577-1587]

38 Feng H J, Chen L G, Wu Z X, et al. Constructal optimization for an organic fluid shell-and-tube condenser based on entransy theory (in Chinese). CIESC J, 2020, 71: 98-103 [冯辉君, 陈林根, 吴志祥, 等. 基于熕理论的有机工质管壳式冷凝器构形优化. 化工学报, 2020, 71: 98-103]

39 Qin X Y, He W S, Zhou G Y. Principle of Marine Boiler (in Chinese). Beijing: National Defense Industry Press, 2016 [秦晓勇, 何文胜, 周国义. 舰用锅炉原理. 北京: 国防工业出版社, 2016]

40 Chen L G, Zhang J M, Wu C, et al. Analysis of multi-objective decision-making for marine steam turbine stage. Int J Power Energy Syst, 1998, 18: $96-101$

41 Feng H J, Chen L G, Liu X, et al. Constructal design for an iron and steel production process based on the objectives of steel yield and useful energy. Int J Heat Mass Transfer, 2017, 111: 1192-1205

42 Cai C G, Feng H J, Chen L G, et al. Constructal design of a shell-and-tube evaporator with ammonia-water working fluid. Int J Heat Mass Transfer, 2019, 135: 541-547

43 Cai C G, Zhao G C, Chen L G, et al. Multi-objecive constructal optimization of a shell-and tube condenser with ammonia-water working fluid (in Chinese). J Eng Thermophys, 2020, 41: 2253-2258 [蔡存光, 赵光超, 陈林根, 等. 基于氨水工质的管壳式冷凝器多目标构形优化. 工程热物 理学报, 2020, 41: 2253-2258]

44 Ahmadi P, Dincer I, Rosen M A. Exergy, exergoeconomic and environmental analyses and evolutionary algorithm based multi-objective optimization of combined cycle power plants. Energy, 2011, 36: 5886-5898

45 Long R, Li B, Liu Z C, et al. Multi-objective optimization of a continuous thermally regenerative electrochemical cycle for waste heat recovery. Energy, 2015, 93: 1022-1029

46 Dai D D, Yuan F, Long R, et al. Performance analysis and multi-objective optimization of a Stirling engine based on MOPSOCD. Int J Thermal Sci, 2018, 124: 399-406

47 Ge Y, Liu Z C, Sun H N, et al. Optimal design of a segmented thermoelectric generator based on three-dimensional numerical simulation and multi-objective genetic algorithm. Energy, 2018, 147: 1060-1069

48 Ge Y, Wang S C, Liu Z C, et al. Optimal shape design of a minichannel heat sink applying multi-objective optimization algorithm and threedimensional numerical method. Appl Thermal Eng, 2019, 148: 120-128

49 Wu Z X, Feng H J, Chen L G, et al. Performance optimization of a condenser in ocean thermal energy conversion (OTEC) system based on constructal theory and a multi-objective genetic algorithm. Entropy, 2020, 22: 641

50 Sun M, Xia S J, Chen L G, et al. Minimum entropy generation rate and maximum yield optimization of sulfuric acid decomposition process using NSGA-II. Entropy, 2020, 22: 1065

51 Shi S S, Ge Y L, Chen L G, et al. Four-objective optimization of irreversible atkinson cycle based on NSGA-II. Entropy, 2020, 22: 1150

52 Chen L G, Tang C Q, Feng H J, et al. Power, efficiency, power density and ecological function optimization for an irreversible modified closed 
variable-temperature reservoir regenerative brayton cycle with one isothermal heating process. Energies, 2020, 13: 5133

54 Tang C Q, Chen L G, Feng H J, et al. Four-objective optimizations for an improved irreversible closed modified simple brayton cycle. Entropy, 2021, 23: 282

55 Chen C, You J, Feng H J, et al. A multi-objective study on the constructal design of non-uniform heat generating disc cooled by radial- and dendritic-pattern cooling channels. Sci China Tech Sci, 2021, 64: 729-744

\title{
Constructal optimization for a marine boiler based on entransy theory
}

\author{
CHEN LinGen ${ }^{1,2}$, FENG HuiJun ${ }^{1,2}$, XIE ZhuoJun ${ }^{3}$, TANG Wei ${ }^{3} \&$ GE YanLin ${ }^{1,2}$ \\ ${ }^{1}$ Institute of Thermal Science and Power Engineering, Wuhan Institute of Technology, Wuhan 430205, China; \\ ${ }^{2}$ School of Mechanical \& Electrical Engineering, Wuhan Institute of Technology, Wuhan 430205, China; \\ ${ }^{3}$ College of Power Engineering, Naval University of Engineering, Wuhan 430033, China
}

Based on entransy theory and constructal theory, performance optimization of a marine boiler is conducted by taking the outer diameters of the third convection evaporation tube, superheater tube, and economizer tube as the design variables with the total heat transfer area constraint of three main heat exchange components (evaporator, superheater, and economizer). The optimal construct and performance of the whole boiler are obtained. First, the optimization is performed by taking a complex function comprising the linear weighted sum of entransy dissipation rate and power consumption rate as the optimization objective. The influences of initial design parameters, such as heat preservation coefficient, weighting coefficient for the composite function, and heat transfer rate, on the constructal optimization results of the whole boiler, are analyzed. Results show that the optimal dimensionless tube outer diameters of the evaporator, superheater, and economizer are 1.2, 1.3, and 1.1, respectively, which make the composite function obtain the cubic minimum value of 0.737 . Compared with the initial design point, the composite function of the overall boiler is reduced by $26.3 \%$, and the comprehensive performance of the boiler is significantly improved. Second, the bi-objective optimization of the overall boiler based on the composite function is conducted based on the NSGA-II algorithm, and the corresponding Pareto frontier and optimal construct of the boiler are obtained, which can provide a wider selection space for the performance optimization and parameter design of the overall boiler. The results of this paper can provide a certain degree of theoretical guidelines for the overall performance optimization design of marine boilers.

entransy theory, constructal theory, marine boiler, entransy dissipation rate, power consumption rate, multiobjective optimization

doi: $10.1360 /$ SST-2021-0054 